\title{
THE CHOW MOTIVE OF SEMISMALL RESOLUTIONS
}

\author{
Mark Andrea A. De Cataldo and Luca Migliorini
}

\begin{abstract}
We show that the topological Decomposition Theorem for a proper semismall map $f: X \rightarrow Y$ implies a "motivic" decomposition theorem for the rational algebraic cycles of $X$ and, in the case $X$ is compact, for the Chow motive of $X$. We work in the category of pure Chow motives over a base. Under suitable assumptions on the stratification, we also prove an explicit version of the motivic decomposition theorem and compute the Chow motives and groups in some examples, e.g. the nested Hilbert schemes of points of a surface. In an appendix with T. Mochizuki, we do the same for the parabolic Hilbert scheme of points on a surface.
\end{abstract}

\section{Introduction}

Borho and MacPherson [3] first realized that the Decomposition Theorem of Beilinson, Bernstein, Deligne and Gabber [2] has a simple and explicit statement for complex proper algebraic semismall maps $f: X \rightarrow Y$ from a manifold. See $\S 2$ for definitions and Theorem 2.2.3.

Semismall maps appear naturally in a variety of contexts arising in geometry and representation theory, e.g. the Springer resolutions of nilpotent cones, maps from Gieseker to Uhlenbeck moduli spaces, Hilbert-Chow maps for various Hilbert schemes of points on surfaces, holomorphic symplectic resolutions, etc. Every stratum for $f$ on $Y$ gives rise to the local system given by the monodromy action on the irreducible components of maximal dimension of the fibers over the stratum. Associated with this local system there is the corresponding intersection cohomology complex on the closure of the stratum.

The Decomposition Theorem for $f$ states that the direct image complex $R f_{*} \mathbb{Q}_{X}[\operatorname{dim} X]$ is isomorphic to the direct sum over the set of relevant strata of these intersection cohomology complexes.

A striking consequence is that, for these maps, the cohomology of the fiber over a point of a stratum gets decomposed in, so to speak, the easiest possible way: all but its top cohomology is a direct sum of the stalks of the cohomology sheaves of the intersection cohomology complexes of the strata "near" the point; the top cohomology comes, naturally, from the stratum itself.

Received January 15, 2002.

M. de Cataldo partially supported by N.S.F. Grants DMS 0202321, DMS 9729992 and by NSA Grant MDA904-02-1-0100.

L. Migliorini partially supported by MIUR project Proprietà Geometriche delle Varietà Reali e Complesse and by GNSAGA. 
This statement is, in a sense, surprising. It is as if the geometry of the fiber did not really count, but only the irreducible components of the fiber and their monodromy along the stratum.

In this paper we show, using the Decomposition Theorem, that given a map $f: X \rightarrow Y$ as above, the picture for the Chow groups and the Chow motive of $X$ is analogous. Here, we freely use the language and properties of correspondences and projectors even if $X$ is not proper, in view of the refined Gysin formalism; see [13] and [8]. In order to do so more efficiently, following a suggestion of the referee, we make use of the category of pure motives over a base. This has been introduced in [6] for smooth varieties. We introduce the analogous notion for $\mathbb{Q}$-varieties. This works well in view of [27] and [28].

We show that new decompositions arise for semismall maps. They imply analogous ones for Chow groups, singular cohomology and mixed Hodge structures.

Every relevant stratum with the associated local system gives rise to a non trivial correspondence in $X \times X$ which is a projector. This correspondence is constructed with the monodromy datum of the irreducible components of the fibers over the stratum.

These projectors are orthogonal to each other. This is the Chow-theoretic and motivic analogue of the fact that each relevant stratum contributes a direct summand in the Decomposition Theorem.

The sum of these projectors is the diagonal of $X$. This corresponds to the fact that the direct summands above add up to the direct image complex $R f_{*} \mathbb{Q}_{X}[\operatorname{dim} X]$.

The precise formulation is given in Theorem 2.3.8 which one may call the Motivic Decomposition Theorem for semismall maps.

In general, even though the formulation of the Decomposition Theorem may be quite explicit, as it is in the case of semismall maps, it may still be difficult in practice to compute the intersection cohomology complexes involved. It is important to know that the decomposition occurs, but it may be difficult to extract information from that fact alone. The geometry of the particular situation is instrumental in making this tool one of the most effective methods of computation in the homology of algebraic maps.

The main result of this paper is that, under suitable hypotheses on the strata, which are verified in many cases of interest, one can recover the algebraic cycles and the motive of $X$ in terms of the corresponding objects for the strata. See Theorem 2.3.8.

Let us state the following easy consequence of Theorem 2.3.8. It is sufficient for many applications and may give the reader a better idea of the results of this paper and of its computational consequences.

Let $f: X \rightarrow Y$ be a semismall map of complex projective varieties with $X$ nonsingular. For every connected relevant stratum $Y_{a}$, let $t_{a}:=(1 / 2)\left(\operatorname{dim} Y_{a}-\right.$ $\operatorname{dim} X), y_{a} \in Y_{a}$ and consider the $\pi_{1}\left(Y_{a}, y_{a}\right)$-set of irreducible maximal dimensional components of $f^{-1}\left(y_{a}\right)$. Let $\nu_{a}: Z_{a} \rightarrow Y_{a}$ be the corresponding not 
necessarily connected covering. Assume that every $Z_{a}$ has a projective compactification $\overline{Z_{a}}$ which is the disjoint union of quotients of nonsingular varieties such that the map $\nu_{a}$ extends to a finite map $\overline{\nu_{a}}: \overline{Z_{a}} \rightarrow \overline{Y_{a}}$. We denote the Chow motive of a nonsingular (or quotient) proper variety $T$ by $[T]$, and the $n$-th twist of it by the Tate motive by $[T](n)$; e.g. $\left[\mathbb{P}^{1}\right] \simeq[p t] \oplus[p t](-1)$. We construct a correspondence $\bar{\Gamma} \subseteq \coprod_{a} \overline{Z_{a}} \times X$ and prove

Theorem 1.0.1. There are natural isomorphisms of Chow motives:

$$
\bar{\Gamma}: \bigoplus_{a}\left[\overline{Z_{a}}\right]\left(t_{a}\right) \simeq[X]
$$

The analogous statement for Chow groups follows easily. The one for Hodge structures, though not new, as it follows from Saito's [25], is given here a direct proof using correspondences, i.e. without relying on the theory of mixed Hodge modules.

We provide the following applications of Theorem 2.3.8.

For every nonsingular complex surface, we determine the Chow groups, the mixed Hodge structures and the Chow motive of Hilbert schemes (Theorem 3.2.1), of nested Hilbert schemes (Theorem 3.3.1) and, in an appendix with T. Mochizuki (§4), of parabolic Hilbert schemes of points. The former is not new; see [8] and also [17]. The second refines Göttsche's result [17]. The third is new.

Our results allow to verify that for some semismall and crepant resolutions of orbifolds, the mixed Hodge structure of the resolution is canonically isomorphic to the corresponding orbifold structure. See $\S 3.4$. This refines the known fact that one had an equality for the corresponding Hodge numbers. See [12] and [29].

The first-named author dedicates this paper to the memory of Meeyoung Kim.

\section{A motivic decomposition theorem for semismall maps}

We work with algebraic varieties over the field of complex numbers and consider Chow groups with rational coefficients. The results of this paper hold, with minor changes, left to the reader, over an algebraically closed field $K$ and in the complex analytic setting. Over the field $K$ one must work with $\mathbb{Q}_{l}$-coefficients, $l \neq \operatorname{char} K$, and with étale homology (cfr. [20]). The results for (parabolic) Hilbert schemes also hold over any field.

2.1. Fibred products of semismall maps. Throughout this section, $f$ : $X \rightarrow Y$ is a proper surjective and semismall morphism of complex algebraic varieties with $n:=\operatorname{dim} X$.

Recall that, in this context, the semismallness of $f$ is equivalent to the following condition: let $\delta \in \mathbb{N}$ and $Y_{f}^{\delta}:=\left\{y \in Y \mid \operatorname{dim} f^{-1}(y)=\delta\right\}$, then $2 \delta \leq \operatorname{dim} Y-\operatorname{dim} Y_{f}^{\delta}, \forall \delta \geq 0$. On the other hand, the smallness of $f$ is equivalent to requiring that $2 \delta<\operatorname{dim} Y-\operatorname{dim} Y^{\delta}, \forall \delta>0$. By checking the semismallness condition on $\delta>0$, one sees immediately that $f$ must be generically finite so that $\operatorname{dim} X=\operatorname{dim} Y=n$.

The following elementary fact plays an important role in what follows. 
Proposition 2.1.1. Let $f^{\prime}: X^{\prime} \rightarrow Y$ and $f: X \rightarrow Y$ be proper maps, semismall over their images. Then

$$
\operatorname{dim} X^{\prime} \times_{Y} X \leq 1 / 2\left(\operatorname{dim} X+\operatorname{dim} X^{\prime}\right) .
$$

Remark 2.1.2. A map $f: X \rightarrow Y$ is semismall if and only if the irreducible components of $X \times_{Y} X$ have dimension at most equal to $\operatorname{dim} X$. Similarly, a map $f: X \rightarrow Y$ is small if and only if the $n$-dimensional irreducible components of $X \times_{Y} X$ dominate $Y$.

Remark 2.1.3. If we assume that $f$ and $f^{\prime}$ are small over their image and $\operatorname{dim} X=\operatorname{dim} X^{\prime}$, then $\operatorname{dim} X \times_{Y} X^{\prime} \leq \operatorname{dim} X=\operatorname{dim} X^{\prime}$ and equality holds if and only if $f(X)=f^{\prime}\left(X^{\prime}\right)$.

By "Thom-Mather Theory," see [15], page 43, we can choose a finite algebraic Whitney stratification for $f: X \rightarrow Y$. By this we mean that, in particular, $Y=\coprod_{b \in B} Y_{b}$, where every space $Y_{b}$ is a locally closed and smooth subvariety of $Y$ and the induced maps $f_{\mid}: f^{-1}\left(Y_{b}\right) \rightarrow Y_{b}$ are locally topologically trivial over $Y_{b}$.

Definition 2.1.4. Let $A:=\left\{a \in B \mid 2 \operatorname{dim} f^{-1}(y)=\operatorname{dim} Y-\operatorname{dim} Y_{a}, \forall y \in Y_{a}\right\}$. We call $A$ the set of relevant strata for $f$.

Remark 2.1.5. While there may be many different stratifications for $f$, the set of subvarieties $\left\{\overline{Y_{a}}\right\}_{a \in A}$ is uniquely determined by $f$.

Remark 2.1.6. A small map has only one relevant stratum, i.e. the dense one.

2.2. The intersection forms and the decomposition theorem for semismall maps. The goal of this section is to define certain intersection numbers which can be defined when $X$ is nonsingular and also when $X$ is a $\mathbb{Q}$-variety.

Definition 2.2.1. A $\mathbb{Q}$-variety is a variety $X$ which is isomorphic, locally in the étale topology, to the quotient of a nonsingular variety $X^{\prime}$ by the action of a finite group $G$ of automorphisms of $X^{\prime}$.

For more details on what follows see [9] and [15].

Let $S$ be a relevant stratum for $f$ and $s \in S$. We have $2 \operatorname{dim} f^{-1}(s)=n-$ $\operatorname{dim} S$. Let $N$ be a contractible euclidean neighborhood of $s$ and $N^{\prime}=f^{-1}(N)$. Let $\left\{F_{i}\right\}$ be the set of irreducible components of maximal dimension $(1 / 2)(n+$ $\operatorname{dim} S)$ of $f^{-1}(S \cap N)$, and let $\left\{f_{i}\right\}$ be the set of irreducible components of maximal dimension $(1 / 2)(n-\operatorname{dim} S)$ of $f^{-1}(s)$. We denote by $f_{i} \cdot F_{j}$ the rational valued refined intersection product defined in $N^{\prime}$; see [13]. It is independent of the choice of $s \in S \cap N$ and is a monodromy invariant, i.e. it is $\pi_{1}(S, s)$-invariant.

We denote Borel-Moore homology (cfr.[5] for example) with rational coefficients by $H^{B M}(-)$. As $s$ varies on $S$, the Borel-Moore classes

$$
\left[f_{i}\right] \in H_{n-\operatorname{dim} S}^{B M}\left(f^{-1}(s)\right)
$$

form a local system $L_{S}^{*}$ and the intersection pairing above forms a pairing $L_{S}^{*} \otimes$ $L_{S}^{*} \rightarrow \mathbb{Q}_{S}$ on this local system. Since the monodromy is finite, $L_{S}^{*}$ is abstractly, i.e. not necessarily via the pairing above, isomorphic to its dual which can be identified with $L_{S}:=\left(R^{n-\operatorname{dim} S} f_{*} \mathbb{Q}_{X}\right)_{\mid S}$. 
For constant coefficients, the Decomposition Theorem of Beilinson, Bernstein, Deligne and Gabber has a simple statement in the case of semismall maps from a $\mathbb{Q}$-variety; see $[9]$.

Given a variety $Z$ and a local system $L$ on a Zariski-dense open subset of its regular part $Z_{r e g}$, we denote by $I C_{Z}(L)$ the Goresky, MacPherson, Deligne intersection cohomology complex associated with $L$. We denote $I C_{Z}\left(\mathbb{Q}_{Z_{\text {reg }}}\right)$ simply by $I C_{Z}$.

Remark 2.2.2. If $Z$ is a $\mathbb{Q}$-variety, then $I C_{Z} \simeq \mathbb{Q}_{Z}[\operatorname{dim} Z]$.

The following is a special case of the Decomposition Theorem of Beilinson, Bernstein, Deligne and Gabber. See [2], [25], [3], [9], [11].

Theorem 2.2.3. Let $f: X \rightarrow Y$ be a proper, semismall algebraic map of algebraic varieties with $X$ a $\mathbb{Q}$-variety. There is a canonical isomorphism

$$
R f_{*} \mathbb{Q}_{X}[n] \simeq_{D_{c c}^{b}(Y)} \bigoplus_{a \in A} I C_{\overline{Y_{a}}}\left(L_{a}\right)
$$

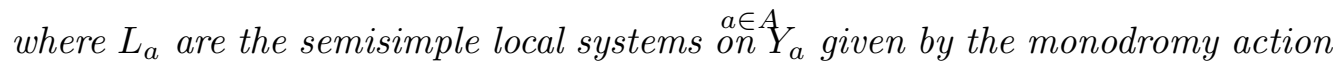
on the maximal dimensional irreducible components of the fibers of $f$ over $Y_{a}$, and $D_{c c}^{b}(Y)$ is the bounded derived category of constructible complexes of sheaves of rational vector spaces on $Y$.

Remark 2.2.4. The Decomposition Theorem is equivalent to the fact that the intersection forms associated with the relevant strata defined in $\S 2.2$ are nondegenerate. In fact, they are definite. See [9] and [11].

2.3. A motivic decomposition for semismall maps. Following [6], we first define the category of pure Chow motives over a base $S$. Note that, according to [27] and [28], the intersection theory formalism developed in [13] for nonsingular varieties can be extended to $\mathbb{Q}$-varieties if we use Chow groups with rational coefficients. In particular, if $Y$ is a $\mathbb{Q}$-variety and $\delta: Y \rightarrow Y \times Y$ is the diagonal imbedding, there exists a refined Gysin map $\delta !$. Given $\phi: Z \rightarrow Y \times Y$, then there is $\delta^{!}: A_{*}(Z) \rightarrow A_{*-\operatorname{dim} Y}\left(Z \times_{Y \times Y} Y\right)$. The Gysin map is in fact defined for the bigger class of Alexander schemes introduced in [27]. However, all the examples we give are $\mathbb{Q}$-varieties, in fact global quotients of nonsingular varieties so that we will stick with $\mathbb{Q}$-varieties.

Let $S$ be an algebraic variety.

Definition 2.3.1. Define a category $\mathcal{C}_{S, \mathbb{Q}}$ as follows. The objects are proper maps $X \stackrel{f}{\rightarrow} S$ from a not necessarily connected $\mathbb{Q}$-variety $X$. Let $Y \rightarrow S$ be an object with $Y$ pure-dimensional. Set

$$
\operatorname{Hom}_{\mathcal{C}_{S, \mathbb{Q}}}(X \rightarrow S, Y \rightarrow S):=A_{\operatorname{dim} Y}\left(X \times_{S} Y\right)
$$

and extend the definition to the case when $Y$ is not equidimensional by additivity.

The composition of morphisms in this category is defined as follows: given $\phi \in A_{\operatorname{dim} Y}\left(X \times_{S} Y\right)=\operatorname{Hom}_{\mathcal{C}_{S, \mathbb{Q}}}(X \rightarrow S, Y \rightarrow S)$ and $\psi \in A_{\operatorname{dim} Z}\left(Y \times_{S} Z\right)=$ $\operatorname{Hom}_{\mathcal{C}_{S, \mathbb{Q}}}(Y \rightarrow S, Z \rightarrow S), \psi \circ \phi$ is defined by means of the fiber product diagram

$$
\begin{array}{rcccc}
X \times_{S} Z & \underset{\downarrow}{p_{X Z}} & X \times{ }_{S} Y \times_{S} Z & \longrightarrow & X \times_{S} Y \times Y \times{ }_{S} Z \\
\text { as } \psi \circ \phi:=p_{X Z *} \delta^{!}(\phi \times \psi) . & Y & \stackrel{\delta}{\longrightarrow} & Y \times Y
\end{array}
$$


Definition 2.3.2. The category $\mathcal{M}_{S, \mathbb{Q}}$ of pure Chow motives over $S$ is defined by adding in the usual way Tate twists to the pseudoabelianization of $\mathcal{C}_{S, \mathbb{Q}}$. See [6] for more details.

An element of $\mathcal{M}_{S, \mathbb{Q}}$ is denoted by $(X \stackrel{f}{\rightarrow} S, P)(r)$ where $P \in A_{\operatorname{dim} X}\left(X \times_{S} X\right)$ is a projector, i.e. $P \circ P=P$ as correspondences, and $r$ is an integer. If $r=0$, then we drop it from the notation and if $P=\Delta_{X}$, then we simply write $X \stackrel{f}{\rightarrow} S$. This latter is called the pure Chow motive of $X$ over $S$.

The notation $[X]$ is reserved for the usual Chow motive $\left(X, \Delta_{X}\right), X$ complete.

Remark 2.3.3. A theorem by B. Toen, [26], shows that the category $\mathcal{M}_{S, \mathbb{Q}}$ is equivalent to the one defined using only proper morphisms $f: X \longrightarrow S$ from a smooth variety $X$. This latter is the definition used in [6].

Remark 2.3.4. a) The category $\mathcal{M}_{\text {Spec } \mathbb{C}, \mathbb{Q}}$ coincides with the classical category of rational Chow motives.

b) If $S$ is a $\mathbb{Q}$-variety, then $H_{\mathcal{M}_{S, \mathbb{Q}}}((X \rightarrow S)(i),(S \stackrel{i d}{\rightarrow} S)(j))=A_{\operatorname{dim} S+i-j}(X)$.

c) There is the contravariant functor $S c h / S \rightarrow \mathcal{M}_{S, \mathbb{Q}}$ which assigns the graph $\Gamma_{f}$ to a $S$-morphism $f: X \rightarrow Y$.

d) Given a proper map $S \rightarrow S^{\prime}$, using composition we obtain a natural covariant functor $\mathcal{M}_{S, \mathbb{Q}} \rightarrow \mathcal{M}_{S^{\prime}, \mathbb{Q}}$. In particular, if $S$ is complete, then $\mathcal{M}_{S, \mathbb{Q}}$ maps to rational Chow motives.

The motives just introduced have a topological realization which is obtained associating functorially with $(X \stackrel{f}{\rightarrow} S)(r)$ the complex $R f_{*} \mathbb{Q}_{X}[2 r]$ in $D_{c c}^{b}(S)$. To define the topological realization at the level of morphisms we need the following

Lemma 2.3.5. Let $f: X \rightarrow S, g: Y \rightarrow S, h: Z \rightarrow S$ be three proper maps of analytic varieties with $X, Y$ and $Z \mathbb{Q}$-varieties and $i, j$ and $k$ be integers. There are canonical isomorphisms

$$
\varphi_{X Y}: \operatorname{Hom}_{D_{c c}^{b}(S)}\left(R f_{*} \mathbb{Q}_{X}[i], R g_{*} \mathbb{Q}_{Y}[j]\right) \simeq H_{2 \operatorname{dim} Y+i-j}^{B M}\left(X \times_{S} Y\right) .
$$

Given morphisms $u: R f_{*} \mathbb{Q}_{X}[i] \rightarrow R g_{*} \mathbb{Q}_{Y}[j], v: R g_{*} \mathbb{Q}_{Y}[j] \rightarrow R h_{*} \mathbb{Q}_{Z}[k]$, one has $\varphi_{X Z}(v \circ u)=\varphi_{Y Z}(v) \bullet \varphi_{X Y}(u)$, where "•" denotes the refined composition of homological correspondences, defined in a way analogous to the definition of composition in $\mathcal{M}_{S, \mathbb{Q}}$.

Proof. [6], Lemma 2.21, Lemma 2.23.

We define the realization for morphisms by means of the diagram:

$$
\begin{aligned}
& \operatorname{Hom}_{\mathcal{M}_{S, \mathbb{Q}}}((X \stackrel{f}{\rightarrow} S)(i),(Y \stackrel{g}{\rightarrow} S)(j)) \simeq \quad A_{\operatorname{dim} Y+i-j}\left(X \times_{S} Y\right) \\
& \operatorname{Hom}_{D_{c c}^{b}(S)}\left(R f_{*} \mathbb{Q}_{X}[2 i], R g_{*} \mathbb{Q}_{Y}[2 j]\right) \simeq H_{2(\operatorname{dim} Y+i-j)}^{B M}\left(X \times_{S} Y\right)
\end{aligned}
$$

where the vertical arrow is the cycle map; see [13], Chapter 19. By Lemma 2.3.5 this map is compatible with the composition of maps in $\mathcal{M}_{S, \mathbb{Q}}$ and $D_{c c}^{b}(S)$. In particular, there are $\mathbb{Q}$-algebra homomorphisms:

$$
\operatorname{End}_{\mathcal{M}_{S, \mathbb{Q}}}(X \stackrel{f}{\rightarrow} S) \longrightarrow \operatorname{End}_{D_{c c}^{b}(S)}\left(R f_{*} \mathbb{Q}_{X}[\operatorname{dim} X]\right) .
$$


Remark 2.3.6. It is also possible to define a mixed Hodge realization of the category $\mathcal{M}_{S, \mathbb{Q}}$ associating with $X \stackrel{f}{\rightarrow} S$ the cohomology groups $H^{*}(X)$ with their mixed Hodge structure. In order to define the realization on morphisms it is necessary to check that the Gysin map defines a morphism of Mixed Hodge structures. This is proved in [10].

Lemma 2.3.7. Let $X \stackrel{f}{\rightarrow} S$ and $Y \stackrel{g}{\rightarrow} S$ be proper maps from $\mathbb{Q}$-varieties, semismall on their images and suppose that $t:=\frac{\operatorname{dim} Y-\operatorname{dim} X}{2}$ is an integer. Then the map:

$\operatorname{Hom}_{\mathcal{M}_{S, \mathbb{Q}}}(X \stackrel{f}{\rightarrow} S,(Y \stackrel{g}{\rightarrow} S)(t)) \rightarrow \operatorname{Hom}_{D_{c c}^{b}(S)}\left(R f_{*} \mathbb{Q}_{X}[\operatorname{dim} X], R g_{*} \mathbb{Q}_{Y}[\operatorname{dim} Y]\right)$ is an isomorphism.

Proof. By Proposition 2.1.1, every component of $X \times{ }_{S} Y$ has dimension at most $(1 / 2)(\operatorname{dim} X+\operatorname{dim} Y)$, so that the cycle map

$$
A_{(1 / 2)(\operatorname{dim} X+\operatorname{dim} Y)}\left(X \times{ }_{S} Y\right) \longrightarrow H_{\operatorname{dim} X+\operatorname{dim} Y}^{B M}\left(X \times{ }_{S} Y\right)
$$

is an isomorphism. The statement follows from Lemma 2.3.5.

Let $X$ be a $\mathbb{Q}$-variety, $f: X \rightarrow Y$ be a proper, surjective and semismall map and $A$ be the set of relevant strata for $f$. Let $a \in A, Y_{a}$ be the corresponding relevant stratum, $t_{a}=(1 / 2)\left(\operatorname{dim} Y_{a}-\operatorname{dim} X\right), y_{a} \in Y_{a}, E_{a}$ be the $\pi_{1}\left(Y_{a}, y_{a}\right)$-set given by the irreducible components of maximal dimension of $f^{-1}\left(y_{a}\right)$ and $\nu_{a}$ : $Z_{a}=\coprod_{i} Z_{a, i} \rightarrow Y_{a}$ be the not necessarily connected étale covering associated with $E_{a}$. For every $a, i$, let $\overline{Z_{a, i}}$ be a $\mathbb{Q}$-variety containing $Z_{a, i}$ as a Zariski-dense open subset and such that there is a proper surjective map $\overline{\nu_{a, i}}: \overline{Z_{a, i}} \rightarrow \overline{Y_{a}}$ extending $\nu_{a, i}:=\nu_{a \mid Z_{a, i}}$, i.e. such that $\overline{\nu_{a, i} \mid Z_{a, i}}=\nu_{a, i}$. Set $\overline{\nu_{a}}: \overline{Z_{a}}=\coprod_{i} \overline{Z_{a, i}} \rightarrow$ $\overline{Y_{a}}$. We will denote by $\overline{\nu_{a}}$ also the composition $\overline{Z_{a}} \stackrel{\overline{\nu_{a}}}{\rightarrow} \overline{Y_{a}} \rightarrow Y$, where the second map is the natural inclusion. Let

$$
R f_{*} \mathbb{Q}_{X}[n] \simeq_{D_{c c}^{b}(Y)} \bigoplus_{a \in A} I C_{\overline{Y_{a}}}\left(L_{a}\right)
$$

be the topological decomposition, and let $P_{a}^{t o p} \in E_{n d_{D_{c c}(Y)}}\left(R f_{*} \mathbb{Q}_{X}[\operatorname{dim} X]\right)$ be the corresponding projectors. By Lemma 2.3.7, they correspond to projectors $P_{a} \in \operatorname{End}_{\mathcal{M}_{Y, \mathbb{Q}}}(X \stackrel{f}{\rightarrow} Y)$. Denote by $I H_{f}$ the projector corresponding to the open stratum of $Y$. Note that if $f$ is generically one-to-one, $I H_{f}^{t o p}$ gives the projection on the Intersection Cohomology complex of $Y$.

Theorem 2.3.8. (Motivic Decomposition Theorem) Let things be as above.

a) There is an isomorphism in $\mathcal{M}_{Y, \mathbb{Q}}$

$$
(X \stackrel{f}{\rightarrow} Y) \simeq \bigoplus_{a}\left(X \stackrel{f}{\rightarrow} Y, P_{a}\right) .
$$

b) If the maps $\overline{\nu_{a, i}}: \overline{Z_{a, i}} \rightarrow \overline{Y_{a}}$ are semismall, then there is an isomorphism in $\mathcal{M}_{Y, \mathbb{Q}}$

$$
\left(X \stackrel{f}{\rightarrow} Y, P_{a}\right) \simeq \bigoplus_{a}\left(\overline{Z_{a}} \stackrel{\overline{\nu_{a}}}{\rightarrow} Y, I H_{\overline{\nu_{a}}}\right)\left(t_{a}\right)
$$


Proof. From the definition of $P_{a}^{t o p}$, we have the equality $I d_{R f_{*} \mathbb{Q}_{X}[n]}=\sum_{a} P_{a}^{t o p}$ in $\operatorname{End}_{D_{c c}^{b}(Y)}\left(R f_{*} \mathbb{Q}_{X}[n]\right)$. By Lemma 2.3.7, this implies the statement a). Similarly, b) is checked by proving the corresponding equality in $\operatorname{End}_{D_{c c}^{b}}(Y)$. The topological realization of $\left(X \stackrel{f}{\rightarrow} Y, P_{a}\right)$ is $I C_{\overline{Y_{a}}}\left(L_{a}\right)[-n]$, the topological realization of $\left(\overline{Z_{a}} \stackrel{\overline{\nu_{a}}}{\rightarrow} Y, I H_{\overline{\nu_{a}}}\right)$ is $I C_{\overline{Y_{a}}}\left(L_{a}\right)\left[-\operatorname{dim} Y_{a}\right]$, whence the statement.

In the discussion of the examples in $\S 3$ and $\S 4$ we will use the following corollary of Theorem 2.3.8:

Corollary 2.3.9. Let things be as in Theorem 2.3.8. Furthermore, assume that the maps $\overline{\nu_{a, i}}: \overline{Z_{a, i}} \rightarrow \overline{Y_{a}}$ are small. Then

$$
(X \stackrel{f}{\rightarrow} Y) \simeq \bigoplus_{a}\left(\overline{Z_{a}} \stackrel{\overline{\nu_{a}}}{\rightarrow} \overline{Y_{a}}\right)\left(t_{a}\right)
$$

Proof. The only $\operatorname{dim} Y_{a}$-dimensional components of $\overline{Z_{a}} \times_{\overline{Y_{a}}} \overline{Z_{a}}$ are dominant, see Remark 2.1.2. It follows that $\left(\overline{Z_{a, i}} \stackrel{\overline{\nu_{a, i}}}{\longrightarrow} \overline{Y_{a}}\right)=\left(\overline{Z_{a, i}} \stackrel{\overline{\nu_{a, i}}}{\longrightarrow} \overline{Y_{a}}, I H_{\overline{\nu_{a, i}}}\right)$.

Corollary 2.3.10. Let things be as in Corollary 2.3.9. Then there exists a correspondence $\bar{\Gamma} \in Z_{*}\left(\coprod_{a} \overline{Z_{a}} \times X\right)$ inducing isomorphisms of Chow groups

$$
\bar{\Gamma}_{*}=\bigoplus_{a \in A} A_{*}\left(\overline{Z_{a}}\right) \longrightarrow A_{*}(X) \quad\left(\text { with } A_{*}\left(\overline{Z_{a}}\right) \longrightarrow A_{*-t_{a}}(X)\right),
$$

and of mixed Hodge structures

$$
\bar{\Gamma}_{*}^{H}=\bigoplus_{a \in A} H^{*}\left(\overline{Z_{a}}\right)\left(t_{a}\right) \longrightarrow H^{*}(X)
$$

2.4. Intersection Chow motives and groups in the presence of semismall resolutions. What follows is a generalization of the construction of Mumford [23] of a (rational valued) intersection product on possibly singular surfaces which we now recall. Let $X$ be a normal surface with a singular point $x$ and let $r: \tilde{X} \rightarrow X$ be a resolution. Denote by $E_{i}$ the exceptional divisors in $r^{-1}(x)$ and by $\Lambda$ the inverse of the intersection matrix $\left(E_{i}, E_{j}\right)$. Given a curve $C$ on $X$, one has a unique lifting to a curve $\tilde{C}$ on $\tilde{X}$ with the condition that $\tilde{C} \cdot E_{i}=0$ for all exceptional curves $E_{i}$. In other words, one is considering the cycle $P=\Delta_{\tilde{X}}-\sum \Lambda_{i, j} E_{i} \times E_{j}$ in $\tilde{X} \times_{X} \tilde{X}$, which is easily seen to be a projector. A direct computation shows that the Chow motive $(X, P)$ has the intersection cohomology of $X$ as its Betti realization.

In a completely analogous way, whenever a singular variety $Y$ is given a semismall resolution $f: X \rightarrow Y$, it is possible to construct a projector $P \in$ $A\left(X \times_{Y} X\right)$ whose Betti realization is the intersection cohomology of $Y$. This construction is meaningful because the Chow motive turns out to be independent of the semismall resolution chosen, as we prove in Proposition 2.4.1. One can therefore talk about intersection Chow motives and groups, in the presence of semismall resolutions.

Let $f: X \rightarrow Y \leftarrow X^{\prime}: f^{\prime}$ be two proper surjective semismall maps, with $X$ and $X^{\prime} n$-dimensional proper nonsingular algebraic varieties. Assume that there 
exists a Zariski-dense open subset $U$ of $Y$ and an isomorphism $g: f^{-1}(U) \rightarrow$ $f^{\prime-1}(U)$ such that $f_{\mid}=f_{\mid}^{\prime} \circ g$ and that $f^{-1}(U) \rightarrow U \leftarrow f^{\prime-1}(U)$ are topological coverings with associated isomorphic local systems $L_{f}$ and $L_{f^{\prime}}$.

The projections $I H_{f}^{t o p}: R f_{*} \mathbb{Q}_{X}[n] \rightarrow I C_{Y}\left(L_{f}\right)=I C_{Y}\left(L_{f^{\prime}}\right) \leftarrow R f_{*}^{\prime} \mathbb{Q}_{X^{\prime}}[n]:$ $I H_{f^{\prime}}^{\text {top }}$ induce, as in the proof of Theorem 2.3.8, algebraic cycles $I H_{f} \in Z_{n}\left(X \times_{Y}\right.$ $X)$ and $I H_{f^{\prime}} \in Z_{n}\left(X^{\prime} \times_{Y} X^{\prime}\right)$ which give Chow motives $\left(X \stackrel{f}{\rightarrow} Y, I H_{f}\right)$ and $\left(X^{\prime} \stackrel{f^{\prime}}{\rightarrow} Y, I H_{f^{\prime}}\right)$, direct summands of $(X \stackrel{f}{\rightarrow} Y)$ and $\left(X^{\prime} \stackrel{f^{\prime}}{\rightarrow} Y\right)$, respectively.

Proposition 2.4.1. Let $f$ and $f^{\prime}$ be as above. There is a canonical isomorphism of pure Chow motives over $Y$

$$
I H_{f^{\prime} f}:\left(X^{\prime} \stackrel{f^{\prime}}{\rightarrow} Y, I H_{f^{\prime}}\right) \rightarrow\left(X \stackrel{f}{\rightarrow} Y, I H_{f}\right)
$$

defined by an algebraic cycle $I H_{f^{\prime} f} \in Z_{n}\left(X^{\prime} \times_{Y} X\right)$.

In addition, if $Y$ is complete, then there is a canonical isomorphism of Chow motives $\left(X, I H_{f}\right) \simeq\left(X^{\prime}, I H_{f^{\prime}}\right)$ each term of which has the intersection cohomology $I H(Y)$ as its Betti realization.

Proof. Let $p_{f^{\prime} f}: R f_{*}^{\prime} \mathbb{Q}_{X^{\prime}}[n] \rightarrow R f_{*} \mathbb{Q}_{X}[n]$ be the map obtained as the composition of the natural maps: $R f_{*}^{\prime} \mathbb{Q}_{X^{\prime}}[n] \rightarrow I C_{Y}\left(L_{f^{\prime}}\right)=I C_{Y}\left(L_{f}\right) \rightarrow R f_{*} \mathbb{Q}_{X}[n]$. Consider the isomorphism of $\mathbb{Q}$-vector spaces

$\psi_{X^{\prime} X}: \operatorname{Hom}_{D_{c c}^{b}(Y)}\left(R f_{*}^{\prime} \mathbb{Q}_{X^{\prime}}[n], R f_{*} \mathbb{Q}_{X}[n]\right) \simeq H_{2 n}^{B M}\left(X^{\prime} \times_{Y} X\right) \simeq Z_{n}\left(X^{\prime} \times_{Y} X\right)$

(see Lemma 2.3.7 and Proposition 2.1.1) and define $I H_{f^{\prime} f}:=\psi\left(p_{f^{\prime} f}\right)$. Analogously, define $p_{f f^{\prime}}$ and $I H_{f f^{\prime}}$. Clearly, $p_{f f^{\prime}} \circ p_{f^{\prime} f}=p_{f^{\prime}}$ and $p_{f^{\prime} f} \circ p_{f f^{\prime}}=p_{f}$. Since $\psi$ is compatible with compositions, see Lemma 2.3.7, the same equations hold for the corresponding cycles, i.e. $I H_{f f^{\prime}} \circ I H_{f^{\prime} f}=I H_{f^{\prime}}$ and $I H_{f^{\prime} f} \circ I H_{f f^{\prime}}=I H_{f}$. The result follows by noting that $I H_{f^{\prime}}$ and $I H_{f}$ induce the identity on the motives $\left(X^{\prime} \stackrel{f^{\prime}}{\rightarrow} Y, I H_{f^{\prime}}\right)$ and $\left(X \stackrel{f}{\rightarrow} Y, I H_{f}\right)$, respectively.

The last statement follows from Remark 2.3.4.d.

Remark 2.4.2. By what above, any two small resolutions of an algebraic variety have canonical isomorphic motives, cycles and mixed Hodge structures. This could also be shown directly using the graph of the corresponding birational map. Remark 2.4.3. If $X \rightarrow Y \leftarrow X^{\prime}$ are two semismall resolutions (in general there is none), then $\left(X \stackrel{f}{\rightarrow} Y, I H_{f}\right)$ and $\left(X^{\prime} \stackrel{f^{\prime}}{\rightarrow} Y, I H_{f^{\prime}}\right)$ are both motivic counterparts of the topological $I C_{Y}$. Proposition 2.4.1 states that they are canonically isomorphic and therefore independent of the semismall resolution.

2.5. Construction of correspondences. In this section we give an explicit description of the correspondences that define the isomorphism of motives stated in Corollary 2.3.9.

We define a set of correspondences $\Gamma_{a, i, I}$ associated with a relevant stratum $Y_{a}$. Their closures define the wanted isomorphisms.

Let $S_{a}$ be a relevant stratum, $s \in S_{a}, E_{a}$ be the right $G_{a}:=\pi_{1}\left(S_{a}, s\right)$-set of maximal dimensional irreducible components of $f^{-1}(s)$. Denote by $\nu_{a}: Z_{a} \rightarrow S_{a}$ the not necessarily connected étale cover of $S_{a}$ corresponding to $E_{a}$. 
By assumption, $Z_{a}$ is a Zariski-dense open subset of a disjoint union of $\mathbb{Q}$-varieties $\overline{Z_{a}}$, and $\nu_{a}: Z_{a} \rightarrow S_{a}$ extends to a small map $\overline{\nu_{a}}: \overline{Z_{a}} \rightarrow \overline{S_{a}} \subseteq Y$. Note that $\overline{Z_{a}}$ is of pure dimension $\operatorname{dim} S_{a}$. The correspondences we will use are supported on the closures in $\overline{Z_{a}} \times_{Y} X$ of $Z_{a} \times_{Y} X$. We introduce notation in order to deal with their top-dimensional irreducible components.

From now on we use the term "component" to refer to a top-dimensional one. Fix representatives $o_{1}, \cdots, o_{r}$ of the $G_{a}$-orbits of $E_{a}$; this corresponds to fixing base points $s_{1}, \cdots, s_{r}$ in the various connected components $Z_{a, 1}, \cdots, Z_{a, r}$ of $Z_{a}$. Let $G_{i}$ be the stabilizer of $o_{i}$ in $G_{a}$. The following is a well-known and elementary fact on $G$-sets:

Lemma 2.5.1. The irreducible components of $Z_{a, i} \times_{Y} X$ are in 1-1 correspondence with the $G_{i}$ orbits of $E_{a}$.

It follows that in order to specify an irreducible component $\Gamma_{a, i, I}$ of $Z_{a, i} \times_{Y} X$ it suffices to specify a $G_{i}$-orbit $I=\left\{f_{i_{1}}, \cdots, f_{i_{l}}\right\}$ of components of $f^{-1}(s)$.

Let $\Gamma_{a, i}$ denote the correspondence $\Gamma_{a, i, o_{i}}$ associated with the $G_{i}$ orbit $\left\{o_{i}\right\}$, $i=1, \ldots r$. Note that the fiber over $s_{i}$ of the natural map $\Gamma_{a, i} \rightarrow Z_{a, i}$ is naturally identified with the corresponding irreducible component of $f^{-1}(s)$ and that $\Gamma_{a, i}$ is an irreducible variety.

Let $\Lambda^{a}=\left(\Lambda_{i, j}^{a}\right)_{i, j \in E_{a}}$ be the inverse of the intersection matrix associated with the pair $\left(S_{a}, s\right)$. Clearly, $\Lambda_{i g, j g}^{a}=\Lambda_{i, j}^{a}, \forall g \in G_{a}$, for the intersection numbers are monodromy invariant. From this follows that, for every $i \in E_{a}, \sum_{j} \Lambda_{i, j}^{a} f_{j}$ is a rational linear combination of $G_{i}$-orbits in the set of maximal dimensional components of $f^{-1}(s)$, and thus defines, by Lemma 2.5.1, a correspondence $\Gamma_{a, i}^{\prime} \in Z\left(Z_{a, i} \times_{Y} X\right)$. Note that in general its support is not irreducible.

Let us summarize what we have done. In general, the pre-image of the stratum $S_{a}$ is not irreducible. In addition, each irreducible component may fail to be irreducible over small euclidean neighborhoods of $s \in S_{a}$. Each irreducible component of the pre-image of a stratum corresponds to a connected étale cover $Z_{a, i}$ and the pull-back $\Gamma_{a, i}$ of this irreducible component to $Z_{a, i}$ is irreducible locally in the euclidean topology over $Z_{a, i}$ and in fact has irreducible fibers over $Z_{a, i}$. The intersection forms being non-degenerate allow to define $\Gamma_{a, i}^{\prime}$.

Define $Z_{a}=\coprod_{i} Z_{a, i}$ and $\overline{\Gamma_{a, i}}$ to be the closure of $\Gamma_{a, i} \subseteq Z_{a, i} \times_{Y} X$ in $\overline{Z_{a, i}} \times_{Y} X$. Similarely for $\overline{\Gamma_{a, i}^{\prime}}$.

Let $\overline{\Gamma_{a}}:=\coprod_{i} \overline{\Gamma_{a, i}}, \bar{\Gamma}:=\coprod_{a} \overline{\Gamma_{a}}, \overline{\Gamma_{a}^{\prime}}:=\coprod_{i} \overline{\Gamma_{a, i}^{\prime}}, \overline{\Gamma^{\prime}}:=\coprod_{a} \overline{\Gamma_{a}^{\prime}}$.

By using the explicit nature of these correspondences, one can easily show the following equality of cycles (proof omitted):

$$
{ }^{t} \overline{\Gamma_{b, j}^{\prime}} \circ \overline{\Gamma_{a, i}}=\delta_{a b} \delta_{i j} \Delta_{\overline{Z_{a, i}} \times \overline{Z_{a, i}}}
$$

from which one can easily deduce that

$$
\bar{\Gamma}: \bigoplus_{a}\left(\overline{Z_{a}} \stackrel{\overline{\nu_{a}}}{\rightarrow} \overline{Y_{a}}\right)\left(t_{a}\right) \simeq(X \stackrel{f}{\rightarrow} Y)
$$

is the isomorphism of Corollary 2.3.9 with inverse ${ }^{t} \overline{\Gamma^{\prime}}$. 


\section{Applications of the Motivic Decomposition Theorem 2.3.8}

We now give a series of applications of Theorem 2.3.8 to the computation of Chow motives and groups; see $\S 3.2, \S 3.3, \S 3.4$ and $\S 4$. It will be sufficient to determine the relevant strata for the maps in question, the fibers over them, and the monodromy on the components of maximal dimension. Except for the situations in $\S 3.4$ and $\S 4$, the fibers are irreducible. This fact alone implies trivial monodromy. Theorem 2.3.8 applies to give the Chow motive and groups of Hilbert schemes and of nested Hilbert schemes. In the former case, that determination had been done in [8] using the same correspondences, but additional knowledge was required, such as the affine cellular structure of the fibers, and the fact that the pre-image of each stratum maps locally trivially over the stratum in the étale topology. By way of contrast, here one only needs to know that the fibers have only one component of maximal dimension. For nested Hilbert schemes, the result is new. The fibers of the maps in $\S 3.4$ are not irreducible, but have trivial monodromy. Finally, the fibers of the Hilbert-Chow map for parabolic Hilbert schemes (cfr. §4) are not irreducible, but they are over the relevant strata and that is enough.

3.1. Maps induced by maps between surfaces. Let $X$ be a nonsingular, connected complex algebraic surface, $Y$ be an algebraic surface and $f: X \rightarrow Y$ be a proper surjective holomorphic map. Note that $f$ is automatically semismall. The map $f$ induces semismall maps $f_{n}: X^{[n]} \rightarrow Y^{(n)}$. We write down the the relevant strata for $f_{n}$ and prove Theorem 3.1.1, an explicit version of Theorem 2.3.8. An application is given in Theorem 3.4.1.

Let $n$ be a positive integer, $X^{[n]}$ the Hilbert scheme of $n$-points of $X$ (cfr. [24], [7]), $S_{n}$ be the $n$-th symmetric group and $X^{(n)}:=X^{n} / S_{n}$ be the $n$-th symmetric product of $X$. There is a natural crepant and semismall map $\pi_{n}: X^{[n]} \rightarrow X^{(n)}$. Let $P(n)$ be the set of partitions $\nu$ of $n$, and let $a=\left(a_{1}, \ldots, a_{n}\right)$, where $a_{i}:=$ the number of times $i$ appears in $\nu$. Clearly, $\sum i a_{i}=n$ and the length $l(a)=$ $l(\nu):=l=\sum a_{i}$.

The morphism $\pi_{n}$ admits a stratification $X^{[n]}=\coprod_{\nu \in P(n)} X_{\nu}^{[n]} \rightarrow \coprod_{\nu \in P(n)} X_{\nu}^{(n)}=$ $X^{(n)}$, where every $X_{\nu}^{(n)}:=\left\{x \in X^{(n)} \mid x=\sum \nu_{k} x_{k}, x_{k} \in X, x_{k} \neq x_{k^{\prime}}\right.$ for $k \neq$ $k^{\prime}$ \}, is a connected locally closed smooth subvariety of $X^{(n)}$ of dimension $2 l(\nu)$, $X_{\nu}^{[n]}:=\left(\pi^{-1}\left(X_{\nu}^{(n)}\right)\right)_{\text {red }}$ and the fibers of $\pi$ over $X_{\nu}^{(n)}$ are irreducible of dimension $n-l(\nu)$.

Let $X^{(\nu)}:=\prod_{i=1}^{n} X^{\left(a_{i}\right)}$. There is a natural map $\pi_{\nu}: X^{(\nu)} \rightarrow X^{(n)}$ which factors through the normalization of $\overline{X_{\nu}^{(n)}} \subseteq X^{(n)}$.

The composition $f_{n}: X^{[n]} \stackrel{\pi_{n}}{\longrightarrow} X^{(n)} \stackrel{f^{(n)}}{\longrightarrow} Y^{(n)}$ is semismall.

A stratification for $f$ induces one for $f^{(n)}$. Using the known one for $\pi_{n}$ in conjunction with the one for $f^{(n)}$ one gets one for $f_{n}$. For our purposes, we need only the stratification by the dimension of the fibers which requires less indices.

Let $V:=Y_{f}^{0} \subseteq Y, U:=f^{-1} V \subseteq X$ and $Y_{f}^{1}:=\left\{y_{1}, \ldots, y_{N}\right\}$. Note that $V$ contains the unique dense stratum for $f$, which is relevant for $f$ and that all the $y_{k}$ are relevant strata, if $Y_{f}^{1} \neq \emptyset$. There are no more relevant strata. 
Let $D_{k}:=\left(f^{-1}\left(y_{k}\right)\right)_{r e d} . D_{k}$ is not necessarily pure-dimensional. Let $H_{k}$ be the set of its one-dimensional components. The one-dimensional part of $D_{k}$ is a not necessarily connected configuration of curves $\left\{C_{k h}\right\}_{h \in H_{k}}$ on $X$.

Let $N \neq 0$. Define, for every $h \geq 0, Q_{N}(h)=\left\{\underline{m}=\left(m_{1}, \ldots, m_{N}\right) \mid m_{k} \in\right.$ $\left.\mathbb{Z}_{\geq 0}, \sum m_{k}=h\right\}$.

If $N=0$, then define $Q_{0}(0)=\{\star\}$ to be a set with one element and $Q_{0}(h)=$ $\emptyset, \forall h>0$.

We have a decomposition

$$
Y^{(n)}=\coprod_{i=0}^{n} \coprod_{\nu \in P(i)} \coprod_{\underline{m} \in Q(n-i)} V_{\nu}^{(i)} \times \star_{\underline{m}}
$$

where $\star$ denotes a point and we identify $V_{\nu}^{(i)} \times \star_{\underline{m}}$ with the locally closed subset of points of type $\sum \nu_{j} v_{j}+\sum_{k=1}^{N} m_{k} y_{k}$.

We have that $f_{n}^{-1}\left(V_{\nu}^{(i)} \times \star_{\underline{m}}\right)=U_{\nu}^{[i]} \times \prod_{k=1}^{N} \pi_{m_{k}}^{-1}\left(D_{k}^{\left(m_{k}\right)}\right)$, where both sides are given the reduced structure.

The reduced fibers over the corresponding stratum for $f_{n}$ are the product of a fiber of $\pi_{i}$ over $X_{\nu}^{(i)}$ with the reduced configuration $\prod_{k=1}^{N} \pi_{m_{k}}^{-1}\left(D_{k}^{\left(m_{k}\right)}\right)$.

Let $\Sigma$ be a finite set and $M_{d}(\Sigma)$ be the set of degee $d$ monomials on the set of variables $\Sigma$.

The first factor above is irreducible and the second has irreducible components of top dimension labeled by $M_{\underline{m}}:=\prod_{k=1}^{N} M_{m_{k}}\left(H_{k}\right)$. The monodromy on these components is trivial.

Consider the natural map $X^{(\nu)}=X_{\underline{m}}^{(\nu)} \rightarrow Y^{(n)}$ obtained by composing $f^{(\nu)}$ with the assignment $\left(z_{1}^{1}, \ldots, z_{a_{1}}^{1} ; z_{1}^{2}, \ldots, z_{a_{2}}^{2} ; \ldots ; z_{1}^{i}, \ldots, z_{a_{i}}^{i}\right) \longrightarrow$ $\sum_{j=1}^{i} \sum_{t=1}^{a_{j}} j z_{t}^{j}+\sum_{k=1}^{N} m_{k} y_{k}$.

Each one of this will count as a relevant stratum as many times as the numbers of irreducible components over it. At this point we can apply Theorem 2.3.8 to $f_{n}$.

Theorem 3.1.1. There are natural isomorphisms of pure Chow motives over $X^{(n)}$

$\bigoplus_{i=0}^{n} \bigoplus_{\nu \in P(i)} \bigoplus_{\underline{m} \in Q(n-i)}\left(X_{\underline{m}}^{(\nu)} \longrightarrow X^{(n)}, P^{(\nu)}\right)^{\oplus\left|M_{\underline{m}}\right|}(i+l(\nu)-2 n) \longrightarrow\left(X^{[n]} \longrightarrow X^{(n)}\right)$

of Chow groups

$$
\bigoplus_{i=0}^{n} \bigoplus_{\nu \in P(i)} \bigoplus_{\underline{m} \in Q(n-i)} P_{*}^{(\nu)}\left(A_{*}\left(X_{\underline{m}}^{(\nu)}\right)\right)^{\oplus\left|M_{\underline{m}}\right|} \simeq A_{*}\left(X^{[n]}\right),
$$

and of mixed Hodge structures

$$
\bigoplus_{i=0}^{n} \bigoplus_{\nu \in P(i)} \bigoplus_{\underline{m} \in Q(n-i)} H^{*}\left(X_{\underline{m}}^{(\nu)}\right)^{\oplus\left|M_{\underline{m}}\right|}((i+l(\nu)-2 n)) \simeq H^{*}\left(X^{[n]}\right) .
$$


If $X$ is assumed to be proper, then there is the isomorphism of Chow motives

$$
\bigoplus_{i=0}^{n} \bigoplus_{\nu \in P(i)} \bigoplus_{\underline{m} \in Q(n-i)}\left(X_{\underline{m}}^{(\nu)}, P^{(\nu)}\right)^{\oplus\left|M_{\underline{m}}\right|}(i+l(\nu)-2 n) \longrightarrow\left[X^{[n]}\right] .
$$

Remark 3.1.2. ((Symmetric) products of semismall maps) Let $f: X \rightarrow$ $Y$ be semismall with $X$ a $\mathbb{Q}$-variety. The induced maps $f^{l}: X^{l} \rightarrow Y^{l}, f^{(l)}$ : $X^{(l)} \rightarrow Y^{(l)}$ and $f^{(\nu)}: X^{(\nu)} \rightarrow Y^{(\nu)}$ are semismall.

We leave to the reader the task of making explicit, using the set of indices $A^{l}$, $A^{(l)}$ and $A^{(\nu)}$, the analogue of Theorem 2.3.8 for the maps $f^{l}, f^{(l)}$ and $f^{(\nu)}$.

3.2. The Hilbert scheme of points on a surface. The following result follows from Corollary 2.3.9:

Theorem 3.2.1. Let $X$ be a nonsingular algebraic surface. There are natural isomorphisms of pure Chow motives over $X^{(n)}$ :

$$
\bar{\Gamma}: \bigoplus_{\nu \in P(n)}\left(X^{(\nu)} \rightarrow X^{(n)}\right)(l(\nu)-n) \simeq\left(X^{[n]} \rightarrow X^{(n)}\right)
$$

inducing isomorphisms of Chow groups

$$
\bar{\Gamma}_{*}: \bigoplus_{\nu \in P(n)} A_{*}\left(X^{(\nu)}\right) \simeq A_{*}\left(X^{[n]}\right)
$$

and of mixed Hodge structures

$$
\bar{\Gamma}_{*}^{H}: \bigoplus_{\nu \in P(n)} H^{*}\left(X^{(\nu)}\right)(l(\nu)-n) \simeq H^{*}\left(X^{[n]}\right) .
$$

If $X$ is assumed to be proper, then there is the isomorphism of Chow motives

$$
\bar{\Gamma}: \bigoplus_{\nu \in P(n)}\left[X^{(\nu)}\right](l(\nu)-n) \simeq\left[X^{[n]}\right] .
$$

Remark 3.2.2. Note that the sign convention in this paper is the opposite of the one in [9].

Remark 3.2.3. Let $A$ be an abelian surface and $K_{n}$ be its $n$-th generalized Kummer variety ([1]). An application of the Corollary 2.3.9 gives a refinement at the level of Chow motives of the formulae for the Betti and Hodge numbers of $A \times K_{n}$ given in [18], Theorems 7 and 8. We omit the details.

3.3. The motive of nested Hilbert schemes of surfaces $X^{[n, n+1]}$. We apply Theorem 2.3.8 to nested Hilbert schemes. The results we obtain refine Göttsche's [17]. Let $X$ be a nonsingular quasi-projective surface, $n$ be a positive integer and define the nested Hilbert scheme of points on $X, X^{[n, n+1]}$, to be the closed subscheme $\{(\eta, \beta) \mid \eta \subseteq \beta\} \subseteq X^{[n]} \times X^{[n+1]}$ given its reduced structure. As is well-known (cfr. [4]), it is connected and smooth of dimension $2 n+2$ and admits a semismall map $\pi_{n, n+1}: X^{[n, n+1]} \rightarrow X^{(n)} \times X$. It is not crepant, e.g. $X^{[1,2]} \rightarrow X \times X$ is the blowing up of the diagonal. This map admits a natural stratification which we now describe.

Fix $\nu \in P(n)$. Define $I_{\nu}=\{0\} \amalg\left\{j \mid a_{j} \neq 0\right\}$. Clearly, $I_{\nu} \backslash\{0\} \neq \emptyset$. 
Define subvarieties $X_{\nu, j} \subseteq X^{(n)} \times X$ as follows

$$
X_{\nu, j}=\left\{\begin{array}{cc}
\left\{(\zeta, x)\left|\zeta \in X_{\nu}^{(n)}, x \notin\right| \zeta \mid\right\} & \text { if } j=0, \\
\left\{(\zeta, x)\left|\zeta \in X_{\nu}^{(n)}, x \in\right| \zeta \mid, \text { length } h_{x}(\zeta)=j\right\} & \text { otherwise. }
\end{array}\right.
$$

The subvarieties $X_{\nu, j} \subseteq X^{(n)} \times X$ are irreducible, smooth, locally closed of dimension $2 l(\nu)+2$, if $j=0$ and $2 l(\nu)$ otherwise. The stratification $X^{(n)} \times X=$ $\coprod_{\nu \in P(n)} \coprod_{j \in I_{\nu}} X_{\nu, j}$ induces, as in $\S 3.1$, a stratification for the map $\pi_{n, n+1}$ for which the fibers are irreducible. The fibers over the stratum $X_{\nu, j}$ have dimension $n-l(\nu)$, if $j=0$ and $n-l(\nu)+1$, if $j \neq 0$. All strata are relevant.

For every $\nu \in P(n)$ and for every $j \in I_{\nu}$, there is a quotient variety $X^{(\nu, j)}$ and a natural generically injective map $X^{(\nu, j)} \rightarrow X^{(n)} \times X$ which factors through the normalization of $\overline{X_{\nu, j}} \subseteq X^{(n)} \times X$, see [4] for details. We are in the position to apply Theorem 2.3.8 and prove the following result

Theorem 3.3.1. Let $X$ be a nonsingular algebraic surface. There are isomorphisms of pure Chow motives over $X^{(n)} \times X$

$$
\bar{\Gamma}: \bigoplus_{\nu \in P(n), j \in I_{\nu}}\left(X^{(\nu, j)} \rightarrow X^{(n)} \times X\right)(m(\nu, j)) \simeq\left(X^{[n, n+1]} \rightarrow X^{(n)} \times X\right),
$$

inducing isomorphisms of Chow groups

$$
\bar{\Gamma}_{*}: \bigoplus_{\nu \in P(n), j \in I_{\nu}} A_{*}\left(X^{(\nu, j)}\right) \simeq A_{*}\left(X^{[n, n+1]}\right),
$$

and of mixed Hodge structures

$$
\bar{\Gamma}^{H}: \bigoplus_{\nu \in P(n), j \in I_{\nu}} H^{*}\left(X^{(\nu, j)}\right)(m(\nu, j)) \simeq H^{*}\left(X^{[n, n+1]}\right)
$$

where $m(\nu, j)=l(\nu)-n$ if $j=0$ and $m(\nu, j)=l(\nu)-n-1$ if $j \neq 0$.

If $X$ is proper, then there is the isomorphism of Chow motives

$$
\bar{\Gamma}: \bigoplus_{\nu \in P(n), j \in I_{\nu}}\left[X^{(\nu, j)}\right](m(\nu, j)) \simeq\left[X^{[n, n+1]}\right] .
$$

3.4. Wreath products, rational double points and orbifolds. Let $G$ be a finite group, $n$ be an integer and $G_{n}$ be the associated Wreath product, i.e. the semidirect product of $G^{n}$ and $S_{n}$. For background on what follows see [30] from which all the constructions below are taken. Let $G \subseteq S L_{2}(\mathbb{C})$ be a finite group. Their classification is known. Let $\tau: \widetilde{\mathbb{C}^{2} / G} \rightarrow \mathbb{C}^{2} / G$ be the minimal and crepant resolution of the corresponding simple singularity. Let $D:=\tau^{-1}(o)$ where $o \in Y$ is the singular point. With the notation of this section, $N=1$, $y_{1}=o$ and the divisor $D$ is supported on a tree of nonsingular rational curves: $|D|=\cup_{k \in H_{1}} C_{k}$.

There is a commutative diagram of semismall and crepant birational morphisms

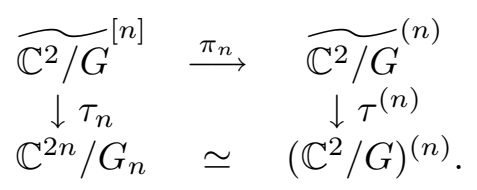


Let $\mathbb{C}_{0}^{2} / G:=\mathbb{C}^{2} / G \backslash\{o\}$. The following is a natural stratification for $\tau_{n}$ for which every stratum is relevant:

$\tau_{n}=\coprod \tau_{n, i, \nu}: \coprod_{i=0}^{n} \coprod_{\nu \in P(i)}\left(\mathbb{C}_{0}^{2} / G\right)_{\nu}^{[i]} \times \pi_{n-i}^{-1}\left(D^{(n-i)}\right) \longrightarrow \coprod_{i=0}^{n} \coprod_{\nu \in P(i)}\left(\mathbb{C}_{0}^{2} / G\right)_{\nu}^{(i)} \times\{(n-i) o\}$.

The fibers over each stratum $\left(\mathbb{C}_{0}^{2} / G\right)_{\nu}^{(i)}$ are isomorphic to the product of the corresponding irreducible fibers of $\pi_{i, \nu}$ with $\pi_{n-i}^{-1}\left(D^{(n-i)}\right)$. The latter factor has dimension $n-i$ and its irreducible components of dimension $n-i$ are naturally labeled by the set $M_{n-i}\left(H_{1}\right)$ of monomials of degree $n-i$ in the set of variables $H_{1}$.

Let $P(i) \ni \nu=\left(a_{1}, \ldots, a_{i}\right)$. The stratum $\left(\mathbb{C}_{0}^{2}\right)_{\nu}^{(i)} \subseteq \prod_{j=1}^{i}\left(\mathbb{C}^{2} / G\right)^{\left(a_{j}\right)}=$ $\prod_{j=1}^{i} \mathbb{C}^{2 a_{j}} / G_{a_{j}}=\mathbb{C}^{2 l(\nu)} / \prod_{j=1}^{i} G_{a_{j}}$. The natural finite map $\mathbb{C}^{2 l(\nu)} / \prod_{j=1}^{i} G_{a_{j}} \rightarrow$ $\left(\mathbb{C}^{2} / G\right)^{(n)}$ factors through the normalization of the closure of the stratum $\left(\mathbb{C}_{0}^{2}\right)_{\nu}^{(i)}$.

Denote by $P=\Delta_{\widetilde{\mathbb{C}}^{2}}-\sum_{h, k} \Lambda_{h, k} C_{h} \times C_{k}$ be the projector corresponding to the projection $R \tau_{*} \underset{\mathbb{Q}_{\mathbb{C}^{2} / G}}{ }[2] \rightarrow I C_{\mathbb{C}^{2} / G} \simeq \mathbb{Q}_{\mathbb{C}^{2} / G}[2]$. Note that one can compute explicitly the projectors $P^{(\nu)}$, for every partition $\nu$ of every integer. We can apply Theorem 2.3.8 and Theorem 3.1.1 and prove

Theorem 3.4.1. There are canonical isomorphisms of Chow groups

$$
\begin{gathered}
\bigoplus_{i=0}^{n} \bigoplus_{\nu \in P(i)} \bigoplus_{M_{n-i}\left(G_{*} \backslash\left\{1^{-}\right\}\right)} A_{*}\left(\mathbb{C}^{2 l(\nu)} / \prod_{j=1}^{i} G_{a_{j}}\right) \simeq \\
\bigoplus_{i=0}^{n} \bigoplus_{\nu \in P(i) M_{n-i}\left(G_{*} \backslash\left\{1^{-}\right\}\right)} P_{*}^{(\nu)} A_{*}\left({\widetilde{\mathbb{C}^{2} / G}}^{(\nu)}\right) \simeq \\
A\left(\widetilde{\widetilde{\mathbb{C}}^{2} / G}{ }^{[n]}\right) \simeq \bigoplus_{\mu \in P(n)} A\left({\widetilde{\mathbb{C}^{2} / G}}^{(\mu)}\right) .
\end{gathered}
$$

Let $G_{*}$ be the set of conjugacy classes of $G$. There is a well-known bijection $b: G_{*} \backslash\left\{1^{-}\right\} \rightarrow T_{G}$ (cfr. [19]).

Using the bijection $b: G_{*} \backslash\left\{1^{-}\right\} \rightarrow T_{G}$ we get a natural set of bijections $M_{n-i}\left(G_{*} \backslash\left\{1^{-}\right\}\right) \rightarrow M_{n-i}\left(T_{G}\right)$.

The set of triplets $(i, \nu, x)$ with $0 \leq i \leq n, \nu \in P(i), x \in M_{n-i}\left(G_{*} \backslash\left\{1^{-}\right\}\right)$is in natural bijection with the set $G_{n *}$ of conjugacy classes of $G_{n}$.

It follows easily that one can identify canonically the orbifold mixed Hodge structure of the orbifold $\mathbb{C}^{2 n} / G_{n}$ with the mixed Hodge structure of $\widetilde{\mathbb{C}}^{2} / G$. The following result refines slightly [29].

Corollary 3.4.2. There is a canonical isomorphism of mixed Hodge structures

$$
\left(H^{*}\left(\mathbb{C}^{2 n} / G_{n}\right)\right)_{\text {orb }} \simeq H^{*}\left({\widetilde{\mathbb{C}^{2} / G}}^{[n]}\right) .
$$

Remark 3.4.3. More generally, let $Y^{\prime}$ be a nonsingular algebraic surface on which a finite group acts as a finite group of automorphism such that the only fixed points are isolated points on $Y^{\prime}$. Let $Y=Y^{\prime} / G$ and $f: X \rightarrow Y$ be its 
minimal resolution, which is automatically crepant. One can prove, using the method above and the analysis of twisted sectors in [29], that the orbifold mixed Hodge structure of $Y^{\prime} n / G_{n}$ is isomorphic to the mixed Hodge structure of $X^{[n]}$. This refines slightly results in [12] and [29].

\section{Appendix: the Chow groups and the Chow motive of parabolic Hilbert schemes; with T. Mochizuki \\ M. de Cataldo, L. Migliorini, T. Mochizuki}

In this appendix we deal explicitely with Chow motives only. See Definition 2.3.2. We do so only because of the lengthy nature of the formulæ. We leave to the reader the task of re-writing the results in terms of pure Chow motives over the base $X^{(n)} \times D^{l_{*}}$.

4.1. The parabolic Hilbert scheme of points on a surface. Let $X$ be an irreducible nonsingular surface defined over an algebraically closed field and $D$ be a nonsingular curve on $X$. Let $I$ be an ideal sheaf on $X$. Recall that a parabolic structure on $I$ of depth $h$ at $D$ is a filtration of the $\mathcal{O}_{D}$-modules, denoted here using the induced successive quotients

$$
I \otimes \mathcal{O}_{D} \longrightarrow \mathcal{G}_{1} \longrightarrow \mathcal{G}_{2} \longrightarrow \cdots \longrightarrow \mathcal{G}_{h} \longrightarrow \mathcal{G}_{h+1}=0 .
$$

An ideal sheaf of points with a parabolic structure is called a parabolic ideal of points. The moduli scheme of the ideal sheaves of points with parabolic structure is called the parabolic Hilbert scheme of points. For simplicity, we only consider the case that $\mathcal{G}_{1}$ is torsion. There is no loss of generality, see [22].

We put the $\mathcal{K}_{i}=\operatorname{Ker}\left(\mathcal{G}_{i} \longrightarrow \mathcal{G}_{i+1}\right)$. For a parabolic ideal sheaf, we denote the length of $\mathcal{K}_{\alpha}$ by $l_{\alpha}$, for $\alpha=1, \ldots, h$. We denote the $h$-tuple $\left(l_{1}, \ldots, l_{h}\right)$ by $l_{*}$. The data $\left(n, h, l_{*}\right)$ is called the type of the parabolic ideal sheaf $\left(I, \mathcal{G}_{*}\right)$.

We denote the parabolic Hilbert scheme of points of $X$ of type $\left(n, h, l_{*}\right)$ by $\operatorname{Hilb}\left(X, D ; n, h, l_{*}\right)$. It is irreducible, nonsingular of dimension $2 n+\sum_{\alpha=1}^{h} l_{\alpha}(\mathrm{cfr}$. [22]). Note that if $l_{\alpha}=0$ for every $\alpha$, then $\operatorname{Hilb}\left(X, D ; n, h, l_{*}\right)$ is isomorphic to $X^{[n]}$.

4.2. The corresponding Hilbert-Chow morphism. For any sheaf $\mathcal{G}$ of finite length, we denote its support with multiplicity by $[\mathcal{G}]$, i.e., if the length of $\mathcal{G}$ at $x$ is $l(x)$, then $[\mathcal{G}]$ defined to be $\sum l(x) \cdot x$. We denote $X^{(n)} \times \prod_{i} D^{\left(l_{i}\right)}$ by $X^{(n)} \times D^{\left(l_{*}\right)}$. We have the natural morphism $F_{n, l_{*}}:$ of $\operatorname{Hilb}\left(X, D ; n, h, l_{*}\right) \rightarrow$ $X^{(n)} \times D^{\left(l_{*}\right)}$ defined by the assignment $\left(I, \mathcal{G}_{*}\right) \longmapsto\left(\left[\mathcal{O}_{X} / I\right],\left[\mathcal{K}_{\alpha}\right]\right)$. We call it the Hilbert-Chow morphism. [22], Corollary 3.1 implies the following

Lemma 4.2.1. The morphism $F_{\left(n, l_{*}\right)}$ is semismall.

Remark 4.2.2. The fibers of $F_{n, l_{*}}$ are not irreducible, in general. However, the ones over relevant strata are; see Lemma 4.3.2. 
4.3. Stratification of the morphism $F_{\left(n, l_{*}\right)}$. We denote the set of integers larger than $i$ by $\mathbb{Z}_{\geq i}$. We put $A=\prod_{\alpha=0}^{h} \mathbb{Z}_{\geq 0}$. An element $v$ of $A$ is described as $v=\left(v_{0}, v_{*}\right)$, where $v_{0}$ is an element of $\mathbb{Z}_{\geq 0}$ and $v_{*}=\left(v_{1}, \ldots, v_{h}\right)$ is an element of $\prod_{\alpha=1}^{h} \mathbb{Z}_{\geq 0}$. We denote the set $A-\{(0, \ldots, 0)\}$ by $A^{\prime}$. We denote the set $\left\{\chi: A^{\prime} \longrightarrow \mathbb{Z}_{\geq 0} \mid \sum_{v} \chi(v)<\infty\right\}$ by $\mathcal{S}\left(A^{\prime}\right)$. We have the natural morphism $\Phi: \mathcal{S}\left(A^{\prime}\right) \longrightarrow \bar{A}$ defined by $\chi \longmapsto \sum_{v} \chi(v) \cdot v$. For any element $u$ of $A$, we put $\mathcal{S}\left(A^{\prime}, u\right):=\Phi^{-1}(u)$.

For any element $\chi \in \mathcal{S}\left(A^{\prime}\right)$, we set:

$$
X_{\chi}:=\prod_{v \in A^{\prime}, v_{*}=0} X^{(\chi(v))} \times \prod_{v \in A^{\prime}, v_{*} \neq 0} D^{(\chi(v))} .
$$

A point of $X_{\chi}$ is denoted $\left(\sum_{k=1}^{\chi(v)} x_{v k} \mid v \in A^{\prime}\right)$, where $x_{v k}$ is a point of $X$ (resp. $D)$ if $v_{*}=0$ (resp. $\left.v_{*} \neq 0\right)$. The dimension of $X_{\chi}$ is $2 \sum_{v_{*}=0} \chi(v)+\sum_{v_{*} \neq 0} \chi(v)$. Let $X_{0 \chi}$ denote the open set of $X_{\chi}$ determined by the condition that $x_{v k} \neq x_{v^{\prime} k^{\prime}}$ for any $(v, k) \neq\left(v^{\prime}, k^{\prime}\right)$.

For any $n \in \mathbb{Z}_{\geq 0}$ and $l_{*} \in \prod_{\alpha=1}^{h} \mathbb{Z}_{\geq 0}$, we have the element $l_{*}(n)=\left(n, l_{*}\right) \in A$. If $\chi$ is contained in $\mathcal{S}\left(A^{\prime}, l_{*}(n)\right)$, we have the finite morphism $G_{\chi}$ of $X_{\chi}$ to $X^{(n)} \times D^{\left(l_{*}\right)}$ defined as follows:

$$
\left(\sum_{k=1}^{\chi(v)} x_{v k} \mid v \in A^{\prime}\right) \longmapsto\left(\sum_{v} \sum_{k=1}^{\chi(v)} v_{\alpha} x_{v k}, \mid \alpha=0,1, \ldots, h\right) .
$$

The restriction of $G_{\chi}$ to $X_{0 \chi}$ is an embedding. We denote the image $G_{\chi}\left(X_{0 \chi}\right)$ also by $X_{0 \chi}$. Then $\left\{X_{0 \chi} \mid \chi \in \mathcal{S}\left(A^{\prime}, l_{*}(n)\right)\right\}$ gives a stratification for the morphism $F_{\left(n, l_{*}\right)}$.

Let $e_{\alpha}$ denote the element of $A^{\prime}$ whose $\beta$-th component is defined to be 1 ( $\alpha=$ $\beta)$ or $0(\alpha \neq \beta)$. We put $C:=\left\{m \cdot e_{0} \mid m \in \mathbb{Z}_{\geq 1}\right\} \cup\left\{m \cdot e_{0}+e_{\alpha} \mid m \in \mathbb{Z}_{\geq 0}, \alpha=\right.$ $1, \ldots, h\}$. The following two lemmata follow from [22], Corollary 3.1.

Lemma 4.3.1. For any point $P \in X_{0 \chi}$, the dimension of the fiber $F_{\left(n, l_{*}\right)}^{-1}(P)$ is $\left(n-\sum_{v_{*}=0} \chi(v)\right)$. The codimension of the stratum $X_{0 \chi}$ is $2\left(n-\sum_{v_{*}=0} \chi(v)\right)+$ $\sum_{\alpha} l_{\alpha}-\sum_{v_{*} \neq 0} \chi(v)$. A stratum $X_{0 \chi}$ is a relevant stratum if and only if $\chi(v)=0$ for any $v$ which is not contained in $C$.

Lemma 4.3.2. If a stratum $X_{0 \chi}$ is relevant, then the top dimensional part of the inverse image $F_{\left(n, l_{*}\right)}^{-1}\left(X_{0 \chi}\right)$ is irreducible.

Let $\overline{\mathcal{S}}\left(A^{\prime}, l_{*}(n)\right)$ denote the subset of $\chi \in \mathcal{S}\left(A^{\prime}, l_{*}(n)\right)$ given by the relevant strata. The set $\overline{\mathcal{S}}\left(A^{\prime}, l_{*}(n)\right)$ can be regarded as the set of $\mathbb{Z}_{\geq 0}$-valued functions $\chi$ on $C$ satisfying $\sum_{v \in C} \chi(v) v=l_{*}(n)$.

Example 4.3.3. $\left(h=1, n=1, l_{1}=1\right)$

In this case, the parabolic Hilbert scheme is isomorphic to the blowing up of $X \times D$ with center $D$, where $D$ is embedded by the composition of the diagonal embedding $D \subset D \times D$ with the natural inclusion $D \times D \subset X \times D$. The HilbertChow morphism is the blowing up morphism. The set $\mathcal{S}\left(A^{\prime},(1,1)\right)$ has two elements: $\delta_{(0,1)}+\delta_{(1,0)}$ and $\delta_{(1,1)}$. Here the function $\delta_{v}: A^{\prime} \longrightarrow \mathbb{Z}_{\geq 0}$ is defined by $\delta_{v}(w)=0(w \neq v)$ and $\delta_{v}(w)=1(w=v)$. In this case $\overline{\mathcal{S}}\left(A^{\prime},(1,1)\right)=$ 
$\mathcal{S}\left(A^{\prime},(1,1)\right)$. The stratum corresponding to $\delta_{(1,0)}+\delta_{(0,1)}\left(\operatorname{resp} . \delta_{(1,1)}\right)$ is $(X \times$ $D \backslash D$ (resp. $D$ ). The dimensions of the fibers corresponding to $\delta_{(1,0)}+\delta_{(0,1)}$ and $\delta_{(1,1)}$ are 0 and 1 , respectively.

Example 4.3.4. $\left(h=1, n=1, l_{1}=2\right)$

In this case, $\mathcal{S}\left(A^{\prime},(1,2)\right)$ has four elements: $\chi_{1}:=\delta_{(1,0)}+2 \delta_{(0,1)}, \chi_{2}:=\delta_{(1,0)}+$ $\delta_{(0,2)}, \chi_{3}:=\delta_{(1,1)}+\delta_{(0,1)}$ and $\chi_{4}:=\delta_{(1,2)}$. The dimensions $d_{i}$ of the fibers and the codimensions $c_{i}$ of the strata corresponding to $\chi_{i}$ are as follows: $\left(d_{1}, c_{1}\right)=$ $(0,0),\left(d_{2}, c_{2}\right)=(0,1),\left(d_{3}, c_{3}\right)=(1,2),\left(d_{4}, c_{4}\right)=(1,3)$. Thus $\overline{\mathcal{S}}\left(A^{\prime},(1,2)\right)$ has two elements $\chi_{1}$ and $\chi_{3}$.

4.4. The results. Let $\bar{\Gamma}$ be the correspondence constructed using the relevant strata above and $\S 2.5$. Let $t_{\chi}=\sum_{v_{*}=0} \chi(v)-n$.

Theorem 4.4.1. We have natural isomorphisms of Chow groups

$$
\bar{\Gamma}_{*}: \bigoplus_{\chi \in \bar{S}\left(A^{\prime}, l_{*}(n)\right)} A_{*}\left(X_{\chi}\right) \longrightarrow A_{*}\left(\operatorname{Hilb}\left(X, D ; n, h, l_{*}\right)\right),
$$

and, if $X$ is assumed to be proper, of Chow motives

$$
\bar{\Gamma}: \bigoplus_{\chi \in \bar{S}\left(A^{\prime}, l_{*}(n)\right)}\left[X_{\chi}\right]\left(t_{\chi}\right) \longrightarrow\left[\operatorname{Hilb}\left(X, D ; n, h, l_{*}\right)\right] .
$$

In what follows, for an algebraic variety $Y, b_{i}(Y)$ denotes the $i$-th Betti number of $Y, P(Y)(z)=\sum_{i} b_{i}(Y) z^{i}$ denotes the Poincaré polynomial of $Y, h^{p, q}(Y)$ denote the $(p, q)$-th Hodge number of $Y, h(Y)(x, y)=\sum_{x, y} h^{p, q}(Y) x^{p} y^{q}$ denotes the Hodge polynomial of $Y$, and we put $\epsilon(p, q)=(-1)^{p+q-1}$.

Corollary 4.4.2. The generating functions for Chow motives (where we denote a motive $\left(T, \Delta_{T}\right)$ simply by $\left.[T]\right)$, Betti and Hodge numbers are

$$
\begin{aligned}
& \sum_{n, l_{*}}\left[\operatorname{Hilb}\left(X, D ; n, h, l_{*}\right)\right] \cdot t^{n} \prod_{\alpha=1}^{h} s_{\alpha}^{l_{\alpha}}= \\
& \prod_{i=1}^{\infty}\left(\sum_{m=0}^{\infty}\left[X^{(m)}\right]((i-1) m) \cdot t^{i m}\right) \times \prod_{\alpha=1}^{h} \prod_{i=0}^{\infty}\left(\sum_{m=0}^{\infty}\left[D^{(m)}\right](i m) \cdot t^{i m} s_{\alpha}^{m}\right) \\
& \sum_{n, l_{*}} P\left(H i l b\left(X, D ; n, h, l_{*}\right)\right)(z) \cdot t^{n} \prod_{\alpha=1}^{h} s_{\alpha}^{l_{\alpha}}= \\
& \prod_{m \geq 1} \frac{\left(1+z^{2 m-1} t^{m}\right)^{b_{1}(X)}\left(1+z^{2 m+1} t^{m}\right)^{b_{3}(X)}}{\left(1-z^{2 m-2} t^{m}\right)^{b_{0}(X)}\left(1-z^{2 m} t^{m}\right)^{b_{2}(X)}\left(1-z^{2 m+2} t^{m}\right)^{b_{4}(X)}} \times \\
& \prod_{\alpha=1}^{h} \prod_{m \geq 0} \frac{\left(1+z^{2 m+1} t^{m} s_{\alpha}\right)^{b_{1}(D)}}{\left(1-z^{2 m} t^{m} s_{\alpha}\right)^{b_{0}(D)}\left(1-z^{2 m+2} t^{m} s_{\alpha}\right)^{b_{2}(D)}} . \\
& \sum_{n, l_{*}} h\left(\operatorname{Hilb}\left(X, D, n, h, l_{*}\right)\right)(x, y) t^{n} \prod_{\alpha=1}^{h} s_{\alpha}^{l_{\alpha}}=
\end{aligned}
$$




$$
\begin{aligned}
& \prod_{m \geq 1} \prod_{0 \leq p, q \leq 2}\left(1+\epsilon(p, q) x^{p+m-1} y^{q+m-1} t^{m}\right)^{\epsilon(p, q) \cdot h^{p, q}(X)} \times \\
& \prod_{\alpha=1}^{h} \prod_{m \geq 0} \prod_{0 \leq p, q \leq 1}\left(1+\epsilon(p, q) x^{p+m} y^{q+m} t^{m} s_{\alpha}\right)^{\epsilon(p, q) \cdot h^{p, q}(D)}
\end{aligned}
$$

Remark 4.4.3. The generating functions for the Betti and Hodge numbers can be found in [22]. The slightly weaker statement in the Grothendieck ring of motives can also be deduced easily from [22].

\section{Acknowledgments}

We thank W. Wang for useful conversations and A.Vistoli for explaining to us the results contained in [27], [28]. We thank A. Corti for stimulating conversations on the subject and for explaining to us the content of the paper [6]. In a certain sense, the main goal of this paper is to show that a small part of the mostly conjectural theory presented in [6] works without relying on the standard conjectures. The first author thanks the Institute for Advanced Study, Princeton, and the Mathematical Sciences Research Institute, Berkeley, for their kind hospitality while this paper was being written. T. Mochizuki, who has coauthored the appendix $\S 4$ wishes to thank the Institute for Advanced Study, Princeton, and to acknowledge N.S.F. Grant DMS 9729992. We also thank the referee for suggesting that we use pure motives over a base in order to streamline our original arguments.

\section{References}

[1] A. Beauville, "Variétés Kähleriennes dont la 1ère classe de Chern est nulle," J.Diff.Geometry, 18 1983, 755-782.

[2] A. Beilinson, J. Bernstein, P. Deligne, Faisceaux pervers, Astérisque 100, (1982).

[3] W. Borho, R. MacPherson, "Partial resolutions of nilpotent varieties," Astérisque 101102 (1983), 23-74.

[4] J. Cheah, "Cellular decompositions for nested Hilbert schemes of points," Pacific J. Math. 183 (1998), 39-90.

[5] N. Chriss, V. Ginzburg, Representation Theory and Complex Geometry, Birkhäuser, Boston Basel Berlin 1997.

[6] A. Corti, M. Hanamura, "Motivic decomposition and intersection Chow groups. I" Duke Math. J. 103 (2000), 459-522.

[7] M. de Cataldo, L. Migliorini, "The Douady space of a complex surface," Adv. in Math. 151, 283-312 (2000).

[8] - "The Chow groups and the motive of the Hilbert scheme of points on a surface," J. of Algebra 251 (2002) no.2, 824-848.

[9] _ "The Hard Lefschetz Theorem and the topology of semismall maps," Ann.Sci. de l'E.N.S. $4^{\text {eme }}$ série, 35 (2002) pp.759-772.

[10] _ "The Gysin map is compatible with mixed Hodge structures," to appear in the Proceedings of "Workshop on algebraic structures and moduli spaces" CRM Université de Montreal.

[11] , "The Hodge Theory of algebraic maps " preprint, AG 0306030 .

[12] J. Bryan, R. Donagi, N. Leung, "G-bundles on abelian surfaces, hyperkähler manifolds, and stringy Hodge numbers," Turkish J. Math. 25 (2001), 195-236. 
[13] W. Fulton, Intersection Theory, Ergebnisse der Mathematik, 3.folge. Band 2, SpringerVerlag, Berlin Heidelberg 1984.

[14] M. Goresky, R. MacPherson, "Intersection homology II," Inv. Math. 71 (1983), 77-129.

[15] _ Stratified Morse Theory, Ergebnisse der Mathematik, 3.folge. Band 2, SpringerVerlag, Berlin Heidelberg 1988.

[16] L. Göttsche, Hilbert schemes of zero-dimensional subschemes of smooth varieties, LNM 1572, Springer-Verlag Berlin 1994.

[17] _ "On the motive of the Hilbert scheme of points on a surface," Meth.Res.Lett. 8 (2001), 613-627.

[18] L. Göttsche, W. Soergel, "Perverse sheaves and the cohomology of the Hilbert schemes of smooth algebraic surfaces," Math. Ann. 296 (1993), 235-245.

[19] Y. Ito, M. Reid, "The McKay correspondence for finite subgroups of $S L(3, \mathbb{C})$," in Higher dimensional complex varieties (Trento, June 1994), (1996), 221-240

[20] G. Laumon, "Homologie étale", Astérisque 36-37 (1976), 163-188.

[21] J. Manin, "Correspondences, motifs and monoidal transformations," Math. USSR-Sb. 6 (1968), 439-470.

[22] T. Mochizuki, "Some topological properties of the parabolic Hilbert schemes," preprint, available at http://www.math.ias.edu/ takuro/list.html.

[23] D. Mumford, "Topology of normal singularities and a criterion for simplicity," Publ. Math. I.H.E.S. 9 (1961), 5-22

[24] H. Nakajima, Lectures on Hilbert schemes of points on surfaces, University Lecture Series, 18 American Mathematical Society, Providence, RI, 1999.

[25] M. Saito, "Decomposition theorem for proper Kähler morphisms," Tohoku Math. J. 42, (1990), 127-147.

[26] B. Toen, "On motives for Deligne-Mumford stacks, "Internat.Math.Res.Notices 17 (2000), 909-928

[27] A. Vistoli, "Alexander duality in intersection theory," Comp. Math. 70, (1989), 199-225.

[28] _ "Intersection theory on algebraic stacks and on their moduli spaces," Invent. Math. 97, (1989), 613-670.

[29] W. Wang, J. Zhou, "Orbifold Hodge numbers of the wreath product orbifolds," J. Geom. Phys. 38 (2001), 152-169.

[30] W. Wang, "Hilbert Schemes, wreath products and the McKay correspondence," math AG 9912104.

Department of Mathematics, SUNy at Stony Brook, Stony Brook, NY 11794, USA

E-mail address: mde@math.sunysb.edu

Dipartimento di Matematica, Università di Bologna, Piazza di Porta S. Donato 5, 40126 BOLOGNA, ITALY

E-mail address: migliori@dm.unibo.it 